\title{
POTENZIALE AUSSCHÖPFEN
}

Liebe Leserin, lieber Leser,

für manche ist es ein Heilsbringer - für manche das Reizwort schlechthin. Die Rede ist von E-Mobilität. Unbestritten ist, dass die Klimaziele 2020 Sinn machen, unstrittig ist auch, dass die konstruktiven Potenziale konsequent ausgeschöpft werden müssen. Man kann daher trefflich streiten, ob die von der Bundesregierung für 2020 ausgelobte Zahl an E-Autos realistisch ist. Wichtig ist allein, dass die E-Technik weiter erforscht, erprobt und effiziente Lösungen auf die Straße gebracht werden.

Konzepte für künftige Elektrofahrzeuge werden sich teilweise deutlich von aktuellen Fahrzeugkonzepten unterscheiden müssen. Radnabenantriebe bieten durch die direkte Anordnung der für den Antrieb notwendigen Komponenten am Rad Vorteile bei Fahrzeugdesign und -konstruktion und eröffnen große Potenziale hinsichtlich Aerodynamik, Raumökonomie, Fahrdynamik sowie passiver und aktiver Sicherheit. Dies kann zu erhöhtem Nutzerkomfort, höheren Reichweiten und damit einer größeren Akzeptanz des Elektroautos auf Kundenseite führen - wenn es gelingt, die hohen Kosten in den Griff zu bekommen. Der vom Fraunhofer LBF prognostizierte Mehrpreis von rund $10 \%$ für ein Fahrzeug mit Radnabenantrieb würde wohl nur dann vom Markt akzeptiert, wenn sich die Preise für E-Fahrzeuge auf ein massentaugliches Maß einpendeln.

Auch Kraft-Wärme-Kopplung ist eines der Zauberwörter für eine ökologisch verantwortungsvolle Zukunft. Im Bereich Haustechnik ist unter anderem Vaillant am Start. In der Kombination mit Mobilität jedoch kamen derartige Systeme, wie etwa die Home Energy Station von Honda, noch nicht über das Stadium des Feldversuchs hinaus. Vor allem die erheblichen Kosten bremsen hier die Markteinführung.
Durchaus vielversprechend ist daher ein Ansatz von fka und ika, bei dem kleine Range-Extender-Module im Fahrzeug helfen, sowohl die mobilitätsbedingten $\mathrm{CO}_{2}-$ Emissionen zu senken als auch beim Anschluss an das Einfamilienhaus dessen Heizsystembedarf positiv zu beeinflussen. Das verbrennungsmotorische Kraftfahrzeug erfüllt so eine Nebenfunktion als (mobile) KWK-Einheit. Die Vorteile liegen vor allem für Plug-in-Hybride und Range-Extender-Konzepte auf der Hand: Die Abwärme der Verbrennungsmaschine dient der Haustemperierung, und der erzeugte Strom kann direkt zur Deckung des aktuellen elektrischen Energiebedarfs genutzt oder aber einer Traktionsbatterie zur Speicherung zugeführt werden.

Alles Zukunftsmusik? Heute vielleicht noch, aber bereits morgen kann diese Technik ihren Siegeszug antreten - wenn ihre Potenziale konsequent ausgeschöpft werden.

Herzlichst Ihr

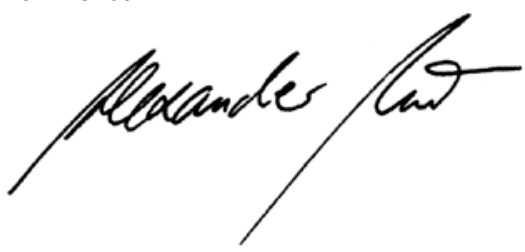

DR. ALEXANDER HEINTZEL, Chefredakteur Wiesbaden, 6. September 2012

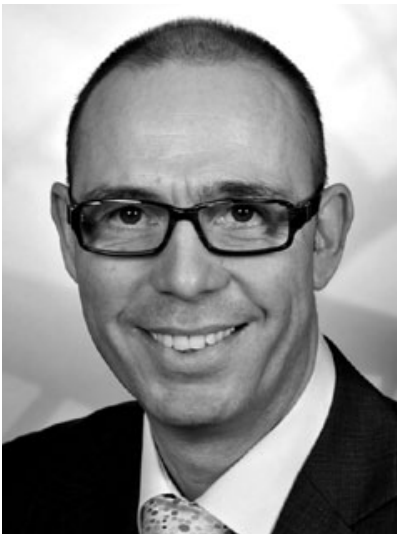

Mit Simulation FreiZeit gewinnen

Thre Zeit-Vorteile

- Umfangreiche Variantenvergleiche durchführen

- Schnell zu ersten Einschätzungen gelangen

- Komplexe Fragestellungen transparent analysieren

\section{Für die Bereiche}

- Fahrleistung und Verbrauch

- Fahrerassistenzsysteme

- Fahrdynamik

- Motordynamik

- Elektro- und Hybridfahrzeuge

Mehr erfahren und am FreiZeit-Gewinnspiel teillnehmen

www.tesis-dynaware.com/izb2012 